\title{
The Use of Point-of-care Testing in Detecting Platelet Function Recovery in a Patient Treated with Prasugrel Undergoing Urgent Surgical Revascularization
}

\author{
Alessandra Verzelloni Sef ${ }^{1}$, Francesca Caliandro ${ }^{1}$, Davorin $\operatorname{Sef}^{1}$, and Shahzad G. Raja ${ }^{1}$ \\ ${ }^{1}$ Affiliation not available
}

September 8, 2021

\section{INTRODUCTION}

Preoperative administration of dual antiplatelet therapy (DAPT) in patients undergoing urgent coronary artery bypass grafting $(\mathrm{CABG})$ surgery remains controversial. DAPT including aspirin and a $\mathrm{P}_{2} \mathrm{Y}_{12}$-inhibitor is most administered before urgent CABG in the setting of acute coronary syndrome (ACS) in accordance with the current guidelines [1]. Although preoperative $\mathrm{P}_{2} \mathrm{Y}_{12}$-inhibitor treatment is associated with reduced occurrence of ischemic events, there is a clear evidence that it can increase the risk of surgery-related bleeding, especially in the case of the third-generation thienopyridines such as prasugrel [2]. Current guidelines recommend a discontinuation of prasugrel a minimum of 7 days before non-emergent cardiac surgery to allow the recovery of platelet function and attenuate the risk of perioperative bleeding [1].

However, these recommendations do not account for highly variable recovery of platelet reactivity following discontinuation of $\mathrm{P}_{2} \mathrm{Y}_{12}$-inhibitor [3]. Prasugrel is an inactive prodrug that is transformed into its active metabolite with a half-life of 7 hours and results in a faster, more consistent platelet inhibition, when compared to clopidogrel $[1,2,4]$. Preoperative point-of-care (POC) platelet function testing (PFT) in patients receiving prasugrel could be helpful to measure platelet reactivity and predict the risk of perioperative bleeding and transfusion requirements [5-8]. We presented a rare case of unexpected complete platelet function recovery in a patient with ACS treated with prasugrel and revealed by preoperative platelet function monitoring with thromboelastography (TEG) platelet mapping before urgent surgical coronary revascularization.

\section{CASE PRESENTATION}

A 59-year-old male presented to the emergency department of a local general hospital with inferior STelevated myocardial infarction (STEMI). The patient had no previous medical history. His vital parameters were stable. After the administration of $300 \mathrm{mg}$ of aspirin and $180 \mathrm{mg}$ of ticagrelor loading dose, eptifibatide infusion was commenced in combination with unfractionated heparin. Emergency angiography revealed severe coronary artery disease $(\mathrm{CAD})$ including long segment disease in mid and distal right coronary artery (RCA), as well as long segment stenosis in the proximal left anterior descending (LAD) artery including ostium of the first diagonal artery. Consecutively, the patient underwent an attempt of PCI to mid and distal RCA, but with suboptimal result due to extensive calcification. Transthoracic echocardiography (TTE) demonstrated a normal-sized left ventricle with a mild systolic impairment. After a 60-mg loading dose of prasugrel, DAPT with aspirin and a 10-mg daily maintenance dose of prasugrel was commenced. The patient was transferred to our tertiary cardiothoracic surgical center for urgent CABG due to the unsuccessful PCI.

At the admission, he remained stable. Troponin I was $625 \mathrm{ng} / \mathrm{L}$ and other blood tests were unremarkable, including platelet count $\left(36710^{\wedge} 9 / \mathrm{L}\right)$. Four days after discontinuation of prasugrel, a preoperative TEG 
PlateletMapping (Haemonetics, Braintree, MA) was performed and ADP-induced platelet aggregation test detected a complete platelet function recovery with maximal platelet aggregation and normal maximum amplitude on TEG (Fig. 1). Therefore, decision was made to proceed with an urgent off-pump CABG which included LIMA-LAD, long saphenous vein to the first diagonal artery and to the posterior descending artery anastomoses. Perioperative blood loss was minimal and no cell salvage or blood product transfusion was required. A loading dose of aspirin $(300 \mathrm{mg})$ was administered within the first 6-24 hours after the surgery and DAPT including $75 \mathrm{mg}$ of both aspirin and clopidogrel was started on the first postoperative day. Postoperative recovery was uneventful and the patient was discharged on the $5^{\text {th }}$ postoperative day and remained well during a 6-month follow-up. Informed consent and patient's permission were obtained to report this case.

\section{DISCUSSION}

Prasugrel has a potent, fast and consistent antiplatelet action and little is known about its impact on platelet function recovery [2]. Perioperative bleeding and blood transfusion requirement can be significantly increased during and after prasugrel therapy [8]. Hereby, we presented a patient with STEMI requiring an urgent $\mathrm{CABG}$ and in whom a complete platelet function recovery after prasugrel was detected by POC testing of platelet function. Despite the initial 60-mg loading dose along with a 10-mg daily maintenance dose over the course of 5 days, complete absence of biological response to prasugrel was demonstrated already 4 days after its discontinuation by preoperative TEG PlateletMapping.

Several factors can be associated with inadequate platelet inhibition by prasugrel such as poor patient compliance, drug absorption disturbances, underdosing and increased platelet turnover [9], while none of these was present in our case. Furthermore, patients with chronic renal failure requiring hemodialysis might have a relatively high rate of prasugrel hyporesponsiveness [10]; however, our patient had normal renal function. On the other hand, Fiore et al. have reported a case of stent thrombosis presenting with recurrent acute onset of chest pain despite being on DAPT including prasugrel and have found that this was related to the drug resistance [9]. Our patient did not have any signs nor predictors of prasugrel resistance. In addition, cytochrome (CYP) variants have been identified in some of the studies as predictors of prasugrel low response [11]. However, it was not possible to perform the genotyping for various CYP single-nucleotide polymorphisms to verify if our patient carried mutant alleles encoding enzymes involved in prasugrel metabolic pathway. In view of the interindividual variability in prasugrel response and platelet function recovery after cessation, we performed a TEG PlateletMapping and we demonstrated a complete recovery of platelet function already on the $4^{\text {th }}$ day after prasugrel discontinuation. By means of POC testing of platelet function, we were able to reduce the waiting time and proceed with surgery.

In a recent randomized double-blinded study, Price et al. found that in aspirin-treated patients with CAD, the greater antiplatelet effect of prasugrel resulted in a more delayed recovery of platelet function as compared with clopidogrel [12]. The authors observed that antiplatelet effects of prasugrel had dissipated in [?]75\% of patients 7 days after its cessation and these findings are consistent with current guidelines regarding the recommended waiting time for surgery after prasugrel discontinuation [12]. In a meta-analysis, Li et al. reported that prasugrel non-response was observed neither at 4 hours following loading dose in 566 patients nor at 9 days in 130 patients following maintenance dose [13]. Furthermore, among patients who underwent CABG in the TRITON-TIMI 38 trial, the rate of CABG-related bleeding was significantly higher with prasugrel compared with clopidogrel, and this increased risk persisted up to 7 days from the most recent dose of study drug [2].

Although current guidelines issue class IIb recommendations for preoperative platelet function assays, the use of preoperative platelet function monitoring to determine the timing of CABG in patients on antiplatelet therapy has been suggested in previous studies [7,14]. Recently, Mannaccio et al. demonstrated the benefits of an individualized preoperative waiting period in patients undergoing off-pump CABG [14]. Importantly, this study challenges current guidelines by showing that a strategy based on individualized POC platelet function measurements resulted in an overall $38 \%$ shortening of the recommended preoperative waiting period for patients on clopidogrel treatment and in a significant reduction of postoperative bleeding and 
blood product consumption.

\section{CONCLUSIONS}

This case report highlights the interindividual variability in recovery time after prasugrel withdrawal and the importance of preoperative PFT in assessing the extent of platelet inhibition in patients who have recently received antiplatelet therapy with prasugrel. We have demonstrated a benefit in the use of preoperative TEG PlateletMapping in a patient undergoing urgent CABG as it can assist in detecting a complete recovery of platelet reactivity and guiding a decision on timing of surgery and optimizing the therapeutic approach without unnecessary blood product transfusion.

Author contributions: AVS,DS: Conceptualization; Investigation; Methodology; Writing-original draft; Writing-Review\&Editing.FC,SGR : Conceptualization; Investigation; Writing-Review\&Editing.

\section{Data Availability Statement}

Data available on request due to privacy/ethical restrictions.

\section{Patient Consent Statement}

The patient agreed to the publication of this case report.

Figure Legends

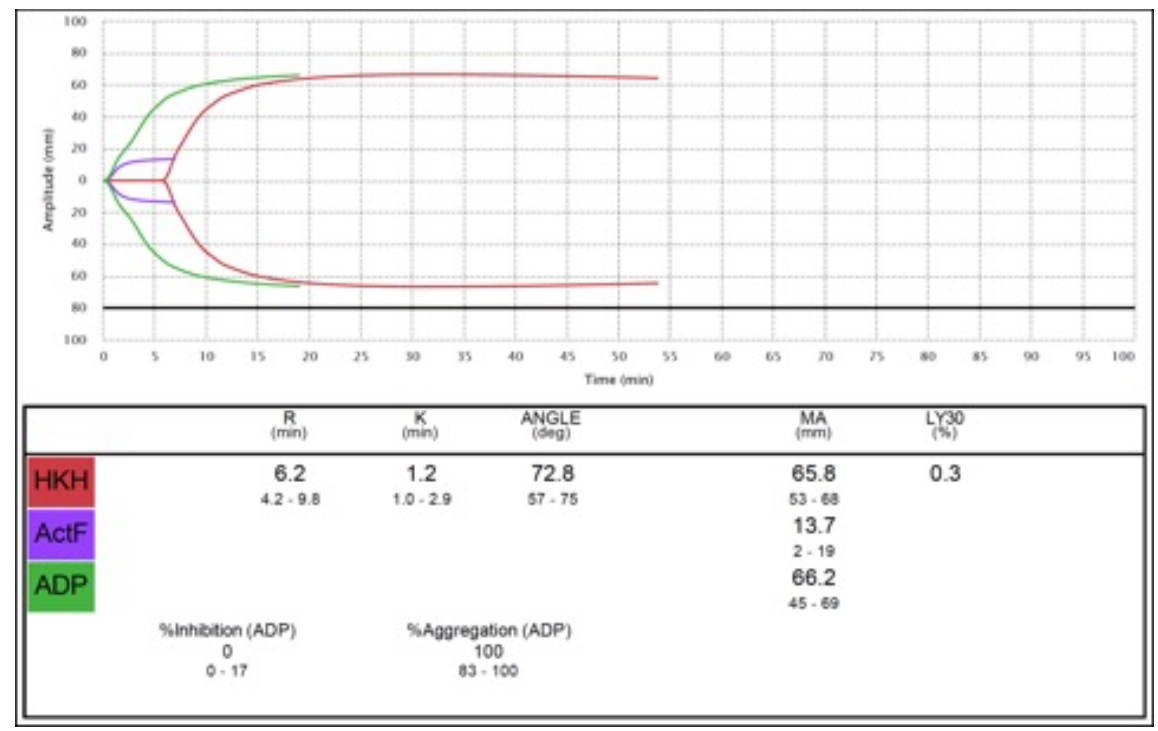

Figure 1: Preoperative TEG PlateletMapping.

Abbreviations: ActF, activator F; ADP, adenosine diphosphate; HKH, kaolin with heparinase.

\section{REFERENCES}

1. Patel MR, Calhoon JH, Dehmer GJ, et al. ACC/AATS/AHA/ASE/ASNC/SCAI/SCCT/STS 2016 Appropriate Use Criteria for Coronary Revascularization in Patients With Acute Coronary Syndromes: A Report of the American College of Cardiology Appropriate Use Criteria Task Force, American Association for Thoracic Surgery, American Heart Association, American Society of Echocardiography, American Society of Nuclear Cardiology, Society for Cardiovascular Angiography and Interventions, Society of Cardiovascular Computed Tomography, and the Society of Thoracic Surgeons. J Am Coll Cardiol 2017;69(5):570-591.

2. Wiviott SD, Braunwald E, McCabe CH, et al. Prasugrel versus clopidogrel in patients with acute coronary syndrome. N Engl J Med 2007;357:2001-15. 
3. Schoergenhofer C, Hobl EL, Staudinger T, et al. Prasugrel in critically ill patients. Thromb Haemost 2017;117(8):1582-1587.

4. Wiviott SD, Antman EM, Braunwald E. Prasugrel. Circulation 2010;122(4):394-403.

5. Yu PJ, Cassiere HA, Dellis SL, Manetta F, Stein J, Hartman AR. P2Y12 platelet function assay for assessment of bleeding risk in coronary artery bypass grafting. J Card Surg 2014;29(3):312-6.

6. Agarwal S. Platelet function testing in cardiac surgery. Transfus Med 2016;26(5):319-329.

7. Rafiq S, Johansson PI, Kofoed KF, Olsen PS, Steinbrüchel DA. Preoperative hemostatic testing and the risk of postoperative bleeding in coronary artery bypass surgery patients. J Card Surg 2016;31(9):565-71.

8. Hansson EC, Jeppsson A. Antiplatelet Therapy, Platelet Function Testing, and Bleeding Complications in Cardiac Surgery Patients. Semin Thromb Hemost 2017;43(7):699-705.

9. Fiore M, Horovitz A, Pons AC, Leroux L, Casassus F. First report of a subacute stent thrombosis in a prasugrel resistant patient successfully managed with ticagrelor. Platelets 2014;25(8):636-8.

10. Alexopoulos D, Panagiotou A, Xanthopoulou I, et al. Antiplatelet effect of prasugrel vs. double clopidogrel in patients on hemodialysis and with high on-treatment platelet reactivity. J Thromb Haemost 2011;9:23792385 .

11. Franken CC, Kaiser AF, Kruger JC, Overbeck K, Mügge A, Neubauer H. Cytochrome P450 2B6 and 2C9 genotype polymorphism - A possible cause of prasugrel low responsiveness. Thrombos Haemost 2013;110:131140.

12. Price MJ, Walder JS, Baker BA, et al. Recovery of platelet function after discontinuation of prasugrel or clopidogrel maintenance dosing in aspirin-treated patients with stable coronary disease: the recovery trial. J Am Coll Cardiol 2012;59:2338-2343.

13. Li YG, Ni L, Brandt JT, Small DS, Payne CD, Ernest CS2 ${ }^{\text {nd }}$, Rohatagi S, Farid NA, Jakubowski JA, Winters KJ. Inhibition of platelet aggregation with prasugrel and clopidogrel: an integrated analysis in 846 subjects. Platelets 2009;20(5):316-27.

14. Mannacio V, Meier P, Antignano A, Di Tommaso L, De Amicis V, Vosa C. Individualized strategy for clopidogrel suspension in patients undergoing off-pump coronary surgery for acute coronary syndrome: a case-control study. J Thorac Cardiovasc Surg 2014;148:1299-1306. 